\title{
Pilot-Scale Batch Alkaline Pretreatment of Corn
}

\section{Stover}

\author{
Erik M. Kuhn ${ }^{1, *}$ Marykate H. O'Brien, ${ }^{1}$ Peter N. Ciesielski, ${ }^{2}$ and Daniel J. Schell ${ }^{1}$ \\ ${ }^{1}$ National Bioenergy Center, National Renewable Energy Laboratory, 16253 Denver \\ West Parkway, Golden, Colorado 80401, United States \\ ${ }^{2}$ Biosciences Center, National Renewable Energy Laboratory, 16253 Denver West \\ Parkway, Golden, Colorado 80401, United States \\ *email: erik.kuhn@nrel.gov
}

Number of Pages: 3

Number of Figures: 0

Number of Tables: 2 
Table S1. Alkaline pretreatment response surface central composite design lignin, xylan, glucan, arabinan, and solids yield data.

\begin{tabular}{|c|c|c|c|c|c|c|c|c|}
\cline { 2 - 9 } \multicolumn{1}{c|}{} & \multicolumn{3}{|c|}{ Factors } & \multicolumn{5}{c|}{ Pretreatment Responses } \\
\hline $\begin{array}{c}\text { standard } \\
\text { order \# }\end{array}$ & $\begin{array}{c}\text { Temperature } \\
\left({ }^{\circ} \mathrm{C}\right)\end{array}$ & $\begin{array}{c}\mathrm{NaOH} \\
(\mathrm{g} / \mathrm{g})\end{array}$ & $\begin{array}{c}\text { AQ }(\%, \\
\mathrm{w} / \mathrm{w})\end{array}$ & lignin (\%) & xylan (\%) & glucan (\%) & $\begin{array}{c}\text { arabinan } \\
(\%)\end{array}$ & solids (\%) \\
\hline 1 & 100 & 40 & 0.05 & $14.9 \%$ & $4.4 \%$ & $1.3 \%$ & $22.6 \%$ & $16.9 \%$ \\
\hline 2 & 140 & 40 & 0.05 & $23.0 \%$ & $11.8 \%$ & $1.6 \%$ & $39.7 \%$ & $24.8 \%$ \\
\hline 3 & 100 & 70 & 0.05 & $48.7 \%$ & $14.0 \%$ & $1.6 \%$ & $45.4 \%$ & $32.6 \%$ \\
\hline 4 & 140 & 70 & 0.05 & $51.5 \%$ & $18.7 \%$ & $1.7 \%$ & $57.5 \%$ & $34.8 \%$ \\
\hline 5 & 100 & 40 & 0.2 & $14.7 \%$ & $4.7 \%$ & $1.3 \%$ & $23.1 \%$ & $16.5 \%$ \\
\hline 6 & 140 & 40 & 0.2 & $25.9 \%$ & $11.3 \%$ & $1.6 \%$ & $37.5 \%$ & $22.7 \%$ \\
\hline 7 & 100 & 70 & 0.2 & $46.5 \%$ & $11.0 \%$ & $1.4 \%$ & $38.8 \%$ & $27.4 \%$ \\
\hline 8 & 140 & 70 & 0.2 & $54.2 \%$ & $16.6 \%$ & $1.3 \%$ & $51.3 \%$ & $33.0 \%$ \\
\hline 9 & 86.36 & 55 & 0.12 & $28.1 \%$ & $6.5 \%$ & $0.6 \%$ & $24.6 \%$ & $21.8 \%$ \\
\hline 10 & 153.64 & 55 & 0.12 & $38.3 \%$ & $18.6 \%$ & $1.4 \%$ & $55.7 \%$ & $31.9 \%$ \\
\hline 11 & 120 & 29.77 & 0.12 & $11.2 \%$ & $4.4 \%$ & $1.6 \%$ & $22.9 \%$ & $16.2 \%$ \\
\hline 12 & 120 & 80.23 & 0.12 & $65.4 \%$ & $17.1 \%$ & $1.7 \%$ & $53.9 \%$ & $36.6 \%$ \\
\hline 13 & 120 & 55 & 0 & $34.2 \%$ & $13.5 \%$ & $1.4 \%$ & $39.8 \%$ & $26.6 \%$ \\
\hline 14 & 120 & 55 & 0.25 & $34.2 \%$ & $13.8 \%$ & $1.4 \%$ & $40.2 \%$ & $27.0 \%$ \\
\hline 15 & 120 & 55 & 0.12 & $35.7 \%$ & $12.9 \%$ & $1.1 \%$ & $42.8 \%$ & $25.0 \%$ \\
\hline 16 & 120 & 55 & 0.12 & $34.2 \%$ & $12.7 \%$ & $1.5 \%$ & $43.1 \%$ & $25.6 \%$ \\
\hline 17 & 120 & 55 & 0.12 & $35.3 \%$ & $13.3 \%$ & $1.4 \%$ & $39.4 \%$ & $26.6 \%$ \\
\hline
\end{tabular}


Table S2. Enzymatic hydrolysis response surface central composite design glucose and xylose yield data.

\begin{tabular}{|c|c|c|c|c|c|c|c|}
\cline { 2 - 8 } \multicolumn{1}{c|}{} & \multicolumn{3}{|c|}{ Factors } & \multicolumn{3}{c|}{ Enzymatic Hydrolysis Responses } \\
\hline $\begin{array}{c}\text { standard } \\
\text { order \# }\end{array}$ & $\begin{array}{c}\text { Temperature } \\
\left({ }^{\circ} \mathrm{C}\right)\end{array}$ & $\begin{array}{c}\mathrm{NaOH} \\
(\mathrm{g} / \mathrm{g})\end{array}$ & $\begin{array}{c}\mathrm{AQ}(\%, \\
\mathrm{w} / \mathrm{w})\end{array}$ & $\begin{array}{c}3 \text { day } \\
\text { glucose } \\
(\%)\end{array}$ & $\begin{array}{c}5 \text { day } \\
\text { glucose } \\
(\%)\end{array}$ & $\begin{array}{c}3 \text { day } \\
\text { xylose }(\%)\end{array}$ & $\begin{array}{c}5 \text { day } \\
\text { xylose }(\%)\end{array}$ \\
\hline 1 & 100 & 40 & 0.05 & $25.4 \%$ & $10.1 \%$ & $33.9 \%$ & $27.9 \%$ \\
\hline 2 & 140 & 40 & 0.05 & $55.6 \%$ & $35.2 \%$ & $69.1 \%$ & $53.9 \%$ \\
\hline 3 & 100 & 70 & 0.05 & $59.3 \%$ & $47.8 \%$ & $73.2 \%$ & $76.8 \%$ \\
\hline 4 & 140 & 70 & 0.05 & $88.5 \%$ & $46.7 \%$ & $96.3 \%$ & $63.9 \%$ \\
\hline 5 & 100 & 40 & 0.2 & $38.2 \%$ & $12.4 \%$ & $47.2 \%$ & $29.0 \%$ \\
\hline 6 & 140 & 40 & 0.2 & $47.9 \%$ & $21.5 \%$ & $61.4 \%$ & $40.0 \%$ \\
\hline 7 & 100 & 70 & 0.2 & $69.5 \%$ & $53.8 \%$ & $80.4 \%$ & $82.6 \%$ \\
\hline 8 & 140 & 70 & 0.2 & $100.0 \%$ & $80.2 \%$ & $100.0 \%$ & $100.0 \%$ \\
\hline 9 & 86.36 & 55 & 0.12 & $33.9 \%$ & $18.7 \%$ & $40.9 \%$ & $38.2 \%$ \\
\hline 10 & 153.64 & 55 & 0.12 & $88.6 \%$ & $70.2 \%$ & $100.0 \%$ & $98.5 \%$ \\
\hline 11 & 120 & 29.77 & 0.12 & $21.6 \%$ & $1.7 \%$ & $34.9 \%$ & $25.6 \%$ \\
\hline 12 & 120 & 80.23 & 0.12 & $90.2 \%$ & $78.9 \%$ & $100.7 \%$ & $100.0 \%$ \\
\hline 13 & 120 & 55 & 0 & $63.8 \%$ & $32.7 \%$ & $79.5 \%$ & $57.2 \%$ \\
\hline 14 & 120 & 55 & 0.25 & $66.6 \%$ & $33.0 \%$ & $81.5 \%$ & $58.0 \%$ \\
\hline 15 & 120 & 55 & 0.12 & $62.6 \%$ & $29.0 \%$ & $63.0 \%$ & $30.5 \%$ \\
\hline 16 & 120 & 55 & 0.12 & $56.9 \%$ & $27.5 \%$ & $69.1 \%$ & $47.4 \%$ \\
\hline 17 & 120 & 55 & 0.12 & $60.9 \%$ & $37.3 \%$ & $73.7 \%$ & $59.8 \%$ \\
\hline
\end{tabular}

